\title{
FY11 Facility Assessment Study for Aeronautics Test Program
}

\author{
John A. Loboda * \\ Jacobs Technology, Tullahoma, TN, 37388 \\ and \\ George H. Sydnor ${ }^{\dagger}$ \\ NASA Langley Research Center, Hampton, VA, 23681
}

This paper presents the approach and results for the Aeronautics Test Program (ATP) FY11 Facility Assessment Project. ATP commissioned assessments in FY07 and FY11 to aid in the understanding of the current condition and reliability of its facilities and their ability to meet current and future (five year horizon) test requirements. The principle output of the assessment was a database of facility unique, prioritized investments projects with budgetary cost estimates. This database was also used to identify trends for the condition of facility systems.

$\begin{array}{ll}A T P & =\text { Aeronautics Test Program } \\ A R C & =\text { Ames Research Center } \\ \text { DoD } & =\text { Department of Defense } \\ F Y & =\text { fiscal year }\end{array}$

\section{Nomenclature}

$$
\begin{array}{ll}
\text { GRC } & =\text { Glenn Research Center } \\
\text { LaRC } & =\text { Langley Research Center } \\
\text { ROM } & =\text { Rough order of magnitude }
\end{array}
$$

\section{Introduction}

$\mathrm{T}$ he NASA Aeronautics Test Program (ATP) is responsible for sustaining a diverse set of valuable aerospace test facilities, located at four NASA Centers across the nation. Maintaining these facilities for safe, reliable, and high-availability operation is required to provide critical high quality research needed by NASA, DoD, and aerospace industry.

To aid in the understanding of the facility condition and reliability and their ability to meet current and future (five year horizon) test requirements, the ATP has established a process of conducting formal periodic facility assessments. An initial facility assessment was performed in FY07? by Jacobs Technology. This initial assessment established a baseline database of recommended near-term and out-year test facility investment projects. The principle output of the assessment was a set of definitized and prioritized projects with budgetary cost estimates. A second assessment was commissioned in FY11 and conducted by an integrated team of multi-discipline engineers from Jacobs

Table 1. ATP Facilities Included in Assessment

Technology and ATP facilities. The FY11 assessment was performed during the period of July 2011 through February 2012 using the same overall techniques

\begin{tabular}{|c|c|}
\hline Langley Research Center (LaRC) & Glenn Research Center (GRC) \\
\hline \multirow{3}{*}{$\begin{array}{l}\text { - National Transonic Facility (NTF) } \\
\text { - } 8 \text {-Ft High Temperature Tunnel (HTT) } \\
\text { - Transonic Dynamics Tunnel (TDT) } \\
\text { - } 14 \text { Ft x } 22 \text { Ft Subsonic Wind Tunnel } \\
\text { - Aerothermodynamics Lab (LAL) } \\
\text { - 31-Inch Mach } 10 \text { Tunnel } \\
\text { - 20-Inch Mach } 6 \text { Hypersonic Tunnel }\end{array}$} & $\begin{array}{l}\text { - Icing Research Tunnel (IRT) } \\
\text { - 10x10 Supersonic Wind Tunnel } \\
\text { - 8x6 and 9x15 Propulsion Wind Tunnel } \\
\text { - Propulsion Systems Lab } 3 \text { and } 4 \text { (PSL) }\end{array}$ \\
\hline & Ames Research Center (ARC) \\
\hline & $\begin{array}{l}\text { - } 11-\text { Ft and 9x7 Unitary Plan Wind } \\
\text { Tunnel (UPWT) }\end{array}$ \\
\hline
\end{tabular}
and tools established in the FY07 assessment. The facilities included in the FY11 assessment are listed in Table 1.

\footnotetext{
* Project Engineer, Jacobs Technology, 600 William Northern Blvd, Tullahoma TN.

${ }^{\dagger}$ NASA Aeronautics Test Program (ATP) Technical Integration Manager, NASA Langley Research Center
} 1 American Institute of Aeronautics and Astronautics 


\section{Assessment Approach}

The FY11 ATP facility assessment was an update to the original (FY08) investment project database. This update included recording the status of the FY08 investment projects and identifying any new projects that would be required in the next five years to ensure availability and operational status of the ATP facilities.

As stated earlier, the assessment team included not only Jacobs engineers but also test engineers from the various ATP facilities. These test engineers, provided valuable insight during the assessment interviews. They also gained valuable working knowledge of all the assessed ATP facilities and were able to establish important relationships with other facility engineers. Many times during the assessment interviews, extremely useful operational and maintenance information was shared between the facility engineers based on current and past experiences.

The flow diagram shown in Fig. 1 depicts the basic process followed for each facility assessment. Prior to each site visit, the assessment team performed the following tasks.

- Established primary point of contact at the facility. This was usually the facility manager.

- Collected background information on the facility (i.e., user manuals, facility handbooks, facility overview presentations, facility schematics, etc.) and familiarized themselves with the facility systems and capabilities.

- Prepared project status update files to document FY08 project statuses and capture new projects. This status update file was sent to the facility manager to update prior to the assessment teams visit.

- Worked with facility manager to establish a date for the assessment that would not interfere with scheduled tests and would ensure that the key facility system engineers and assessment team

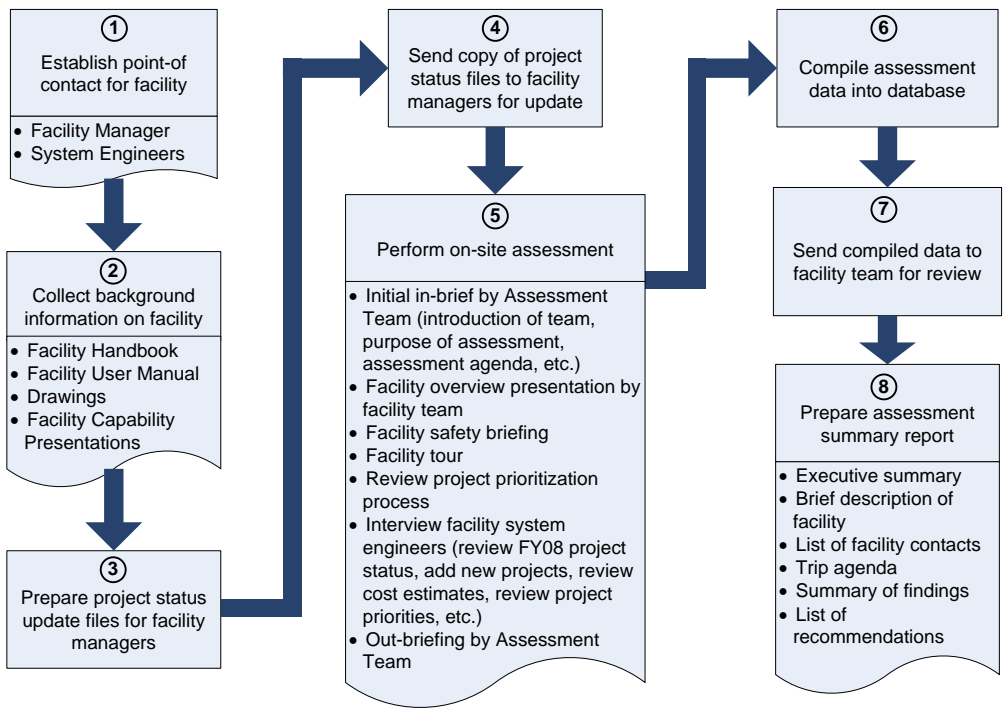

Figure 1. Facility Assessment Process members would be available to support the assessment.

During the site visit, the following activities were accomplished.

- Attended a facility overview briefing by the facility manager. During this meeting, a facility safety briefing was given to the assessment team.

- The ATP manager reviewed the purpose of the assessment, and the assessment project manager reviewed the assessment process and agenda.

- Performed an extensive facility tour during which the team "walked down" the major facility systems, subsystems, and critical components

- Conducted interviews with the facility management, operations and maintenance personnel to collect the necessary facility assessment data. Established priorities for each identified project. Established an understanding of project scope in order to prepare a rough order of magnitude (ROM) cost estimate. Collected any available cost estimates from facility personnel. The data was recorded on the assessment spreadsheets and conversations were recorded using a digital voice recorder.

- Collected other relevant documentation (i.e., photographs, drawings, quotes, investment project lists, etc.)

During the assessment visits, the project database was reviewed and updated live during the interview. Much care was taken to ensure the facility team was comfortable with the projects identified and understood and agreed with the project priorities assigned.

Upon completion of each site visit, the assessment team returned to the home office to compile the collected data. With the compiled facility data, the assessment team produced a prioritized list of recommended investment projects and prepared a budget planning cost estimates for each project. The investment project list and cost 
estimates were then sent to the facility manager for their review and comments. Any comments or changes received were reviewed and incorporated.

ROM cost estimates, for budget planning, were developed for each investment project. Some of the cost estimates were provided by the facility engineers from actual quotes received from vendors or from the local Center engineering support group. Other estimates were based on the assessment team's best understanding of the preliminary scope of work for each recommended project. All estimates include both material and labor hour costs required to complete the project.

One of key outputs of this assessment was a prioritized list of recommended investment projects. To prioritize each project, a risk to test matrix was created (Fig. 2). The priorities ranged from a Priority 1 (highest risk-to-test or injury) to a Priority 5 (lowest risk). The project priorities were assigned for each individual facility (no relation to other ATP facilities) and only addressed facility infrastructure and not address impacts to specific NASA missions (assumes facility is required to support continuous tests over the next five years).

\begin{tabular}{|c|c|c|c|c|c|c|}
\hline \multirow[b]{2}{*}{ Risk Matrix } & & \multicolumn{5}{|c|}{ Probability of Occurrence } \\
\hline & & $\begin{array}{l}\text { Unlikely to } \\
\text { Occur }\end{array}$ & $\begin{array}{l}\text { Could occur } \\
\text { in next } 5 \\
\text { years } \\
\text { (probability } \\
<35 \% \text { ) }\end{array}$ & $\begin{array}{l}\text { Likely to } \\
\text { occur within } \\
\text { next } 5 \text { years } \\
\text { (probability } \\
>35 \% \text { ) }\end{array}$ & $\begin{array}{l}\text { Likely to } \\
\text { occur within } \\
\text { next } 12 \\
\text { months }\end{array}$ & $\begin{array}{c}\text { Existing } \\
\text { chronic } \\
\text { condition or } \\
\text { history of } \\
\text { occurring more } \\
\text { than once per } \\
\text { year } \\
\end{array}$ \\
\hline Consequence of Occurrence & & 1 & 2 & 3 & 4 & 5 \\
\hline $\begin{array}{l}\text { - More than } 6 \text { months of lost test time } \\
\text { - Loss of life or permanent disabling condition }\end{array}$ & 5 & 4 & 2 & 2 & 1 & 1 \\
\hline $\begin{array}{l}\text { - } 1 \text { to } 6 \text { months of lost test time/reduced test } \\
\text { capability } \\
\text { - Severe occupational illness }\end{array}$ & 4 & 4 & 3 & 2 & 1 & 1 \\
\hline $\begin{array}{l}\text { - } 1 \text { month of lost test time/reduced test } \\
\text { capability } \\
\text { - Minor injury occupational injury or illness }\end{array}$ & 3 & 4 & 3 & 3 & 2 & 2 \\
\hline $\begin{array}{l}\text { - } 1 \text { week of lost test time/reduced test capability } \\
\text { - Addition or restoration of facility capability } \\
\text { required for future test requirements } \\
\text { - Significantly reduces cost to operate } \\
\text { - Minor first aid treatment }\end{array}$ & 2 & 5 & 4 & 4 & 3 & 3 \\
\hline $\begin{array}{l}\text { - No impact on lost test time/reduced test } \\
\text { capability } \\
\text { - Desired capability addition / improvement, or } \\
\text { - Improved working environment } \\
\text { - Unlikely to lead to an injury }\end{array}$ & 1 & 5 & 5 & 4 & 4 & 4 \\
\hline
\end{tabular}

Figure 2. Risk to Test Matrix

A large number of projects (668) were identified during the ATP assessment which addressed many different types of facility systems. To obtain an overview of the facility condition and to identify project trends, a method for organizing the projects was needed. This was accomplished by establishing a standard set of facility system categories to which all projects, no matter what type of facility, could be assigned. This method of grouping allowed the data to be easily sorted and plotted to provide insight into inherent trends. The system categories used for this assessment and a brief description for each is presented below.

Safety: Projects included in this category primarily address personnel safety issues related to facility configuration rather than condition-induced safety issues. For example, a project to install fall protection where none had previously existed would be included in this section. However, a project to address a system that has deteriorated due to age and represented a risk to operations as well as personnel and property safety would typically be included in one of the other system categories. 
Electrical Power: This category covered locally-dedicated power distribution systems that supplies power to the facility and systems that regulate, control, or condition power within the facility.

Data Acquisition Systems: Facility data acquisition, processing, and analysis systems are assigned to this category and include instrumentation and sensors, signal conditioning hardware and software, data collection and data processing software, and data storage systems.

Control Systems: This category includes control systems for facility, test support system, and test article control systems such as programmable logic controllers, relay-logic control systems, Control communication systems, computers, controls software, instrumentation and sensors, and human-machine interface hardware and software Test Facility Structures: This category includes projects that address the facility structure such as tunnel ducting, facility supports structures, foundations, and painting.

Cooling Water Systems: Projects in this category address systems and components such as cooling tower systems, piping, valves, pumps, deionized water systems, and heat exchangers.

Test Support Systems: This category includes general facility subsystems that directly support test operations such as tunnel nozzle systems, model injection/positioning and support systems, external balances systems, makeup air systems, imaging systems, and hydraulic systems.

Cryogenic Systems: Several facilities included in this study have cryogenic systems (i.e., liquid nitrogen, and oxygen). Typical cryogenic systems and components included in this category are valves, pumps, storage tanks, piping, and heat exchangers.

Vacuum Systems: This category covers both electromechanical and ejector type vacuum systems and includes components and systems such as steam or air ejector systems, compressors, pumps, blowers, piping/ducting and valves

Test Gas Systems: This category includes systems that are used to provide and/or condition the facility test gases such as gas refrigeration systems, gas heaters, heat exchangers, air dryers, high pressure gas systems and compressors systems.

Facility Fan/Compressor Systems: This category includes tunnel main drive and compressors systems, lube-oil systems, turning gear, gear boxes, and health monitoring systems.

General Building: Included in this category are building systems and structures that are critical to facility operations such as roofs, HVAC, and lighting. An example of a critical facility operations building systems would be a control room air conditioner system or the leaking roof over a data acquisition room.

\section{Individual Facility Assessment Results}

The recommended investment project data was compiled and reported for each facility. Due to the large quantity of assessment data, this report will only present an example of the facility data reported in the assessment final report ${ }^{1}$.

The data presented for each facility consisted of the following tables and figures.

Project Priority Total Cost by Facility Subsystems: This data presents a summary of facility projects costs sorted by system category and priority. An example of the LaRC 8-Ft High Temperature Tunnel (HTT) project summary data is presented in Table 2, Fig. 3, and Fig. 4. 
Table 2. FY11 LaRC 8-Ft HTT Project Priority Total Cost (\$K) by System Category

\begin{tabular}{|r|c|c|c|c|c|c|}
\hline System Category & $\begin{array}{c}\text { Priority } \\
\mathbf{1}\end{array}$ & $\begin{array}{c}\text { Priority } \\
\mathbf{2}\end{array}$ & $\begin{array}{c}\text { Priority } \\
\mathbf{3}\end{array}$ & $\begin{array}{c}\text { Priority } \\
\mathbf{4}\end{array}$ & $\begin{array}{c}\text { Priority } \\
\mathbf{5}\end{array}$ & Total \\
\hline Safety & & & & 210 & & 210 \\
\hline Electrical Power & & & 585 & & 115 & 700 \\
\hline Data Acquisition Systems & & & & 130 & & 130 \\
\hline Control Systems & & 480 & 1,105 & 40 & & 1,625 \\
\hline Cest Facility Structures & & & & & & \\
\hline Test Support Systems & & 50 & 40 & 125 & & 215 \\
\hline Cryogenic Systems & & & 340 & & & 340 \\
\hline Vacuum Systems & & & & & \\
\hline Test Gas Systems & & 485 & & 455 & & 940 \\
\hline Facility Fan/Compressor Systems & & & & & \\
\hline General Building & & & 45 & & 110 & 155 \\
\hline Total & & $\mathbf{1 , 0 1 5}$ & $\mathbf{2 , 1 1 5}$ & $\mathbf{9 6 0}$ & $\mathbf{2 2 5}$ & $\mathbf{4 , 3 1 5}$ \\
\hline
\end{tabular}

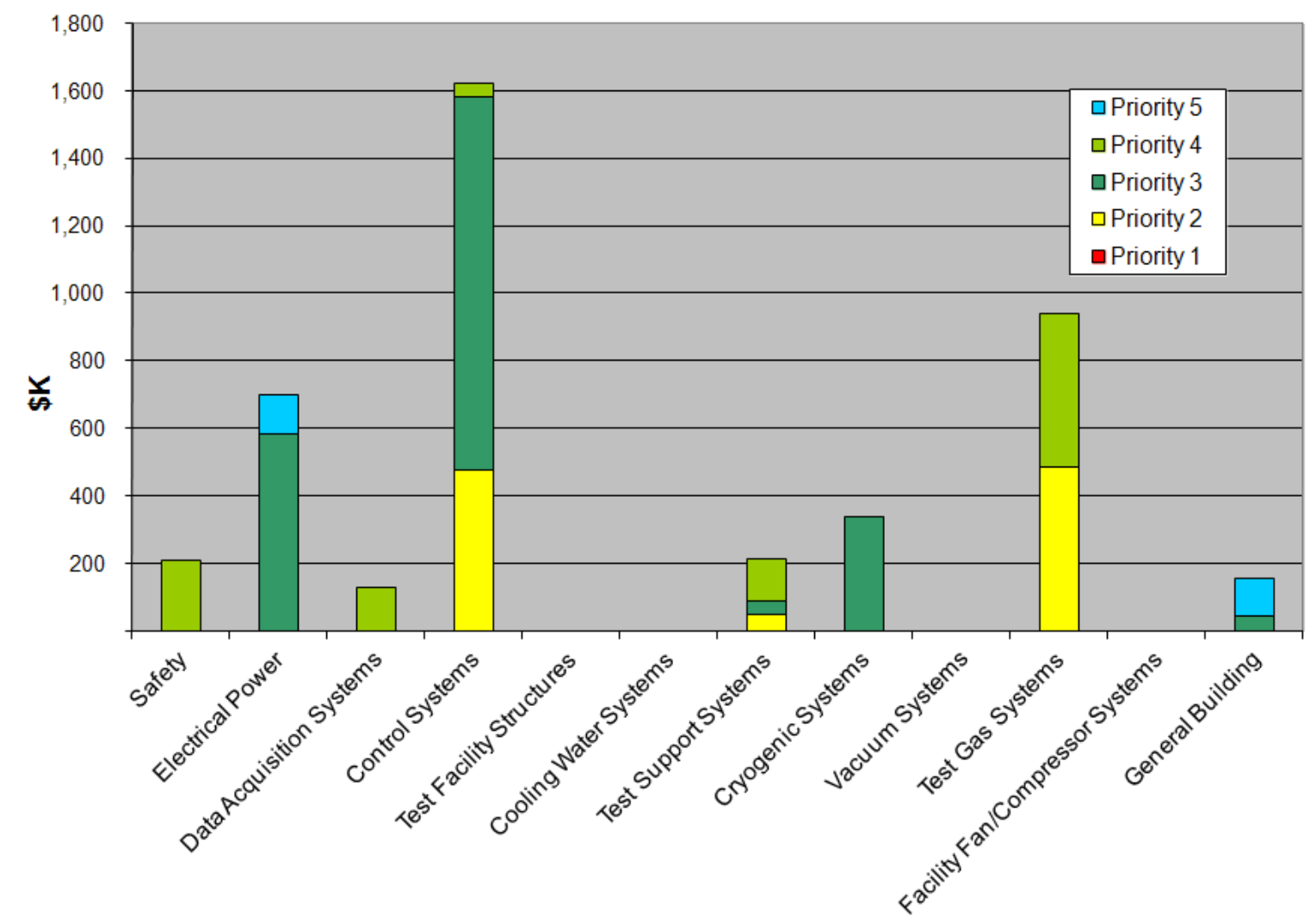

Figure 3. FY11 LaRC 8-Ft HTT Facility Total Investment Costs by System Category 


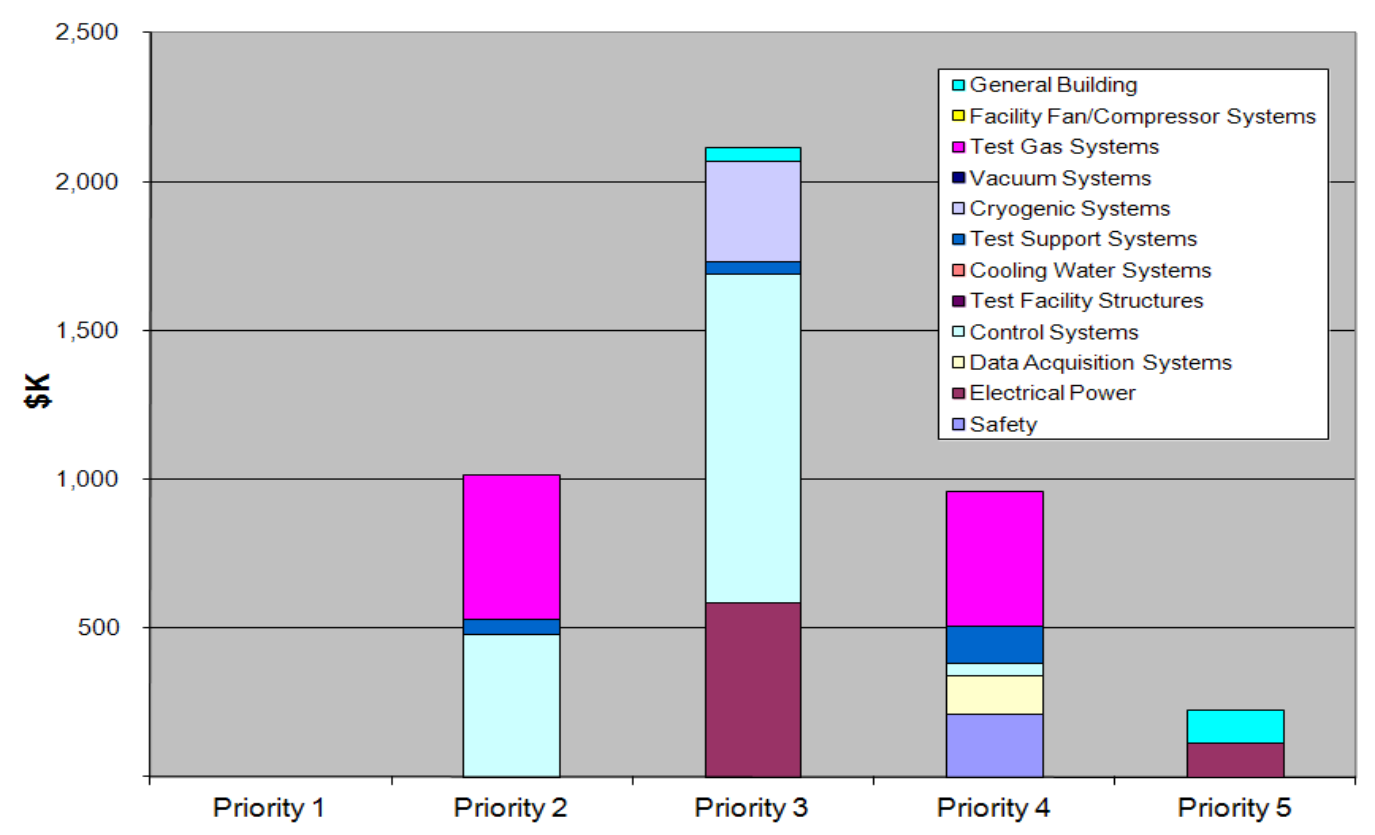

Figure 4. FY11 LaRC 8-Ft HTT Facility Total Investment Costs By Priority

Recommended Investment Projects List- This list (Table 3) includes the following information.

- Brief project description sorted by system category

- Estimated ROM Cost: The budget planning cost estimate for each project includes subtotals for each system category.

- $\quad$ Risk: This section includes the following risk information for each project.

$\circ$ Severity and probability scores

- Priority (1 High Priority through 5 Low Priority)

- Priority Costs: This is a summary of project costs sorted by priority.

Table 3. FY11 LaRC 8-Ft HTT Assessment Project List

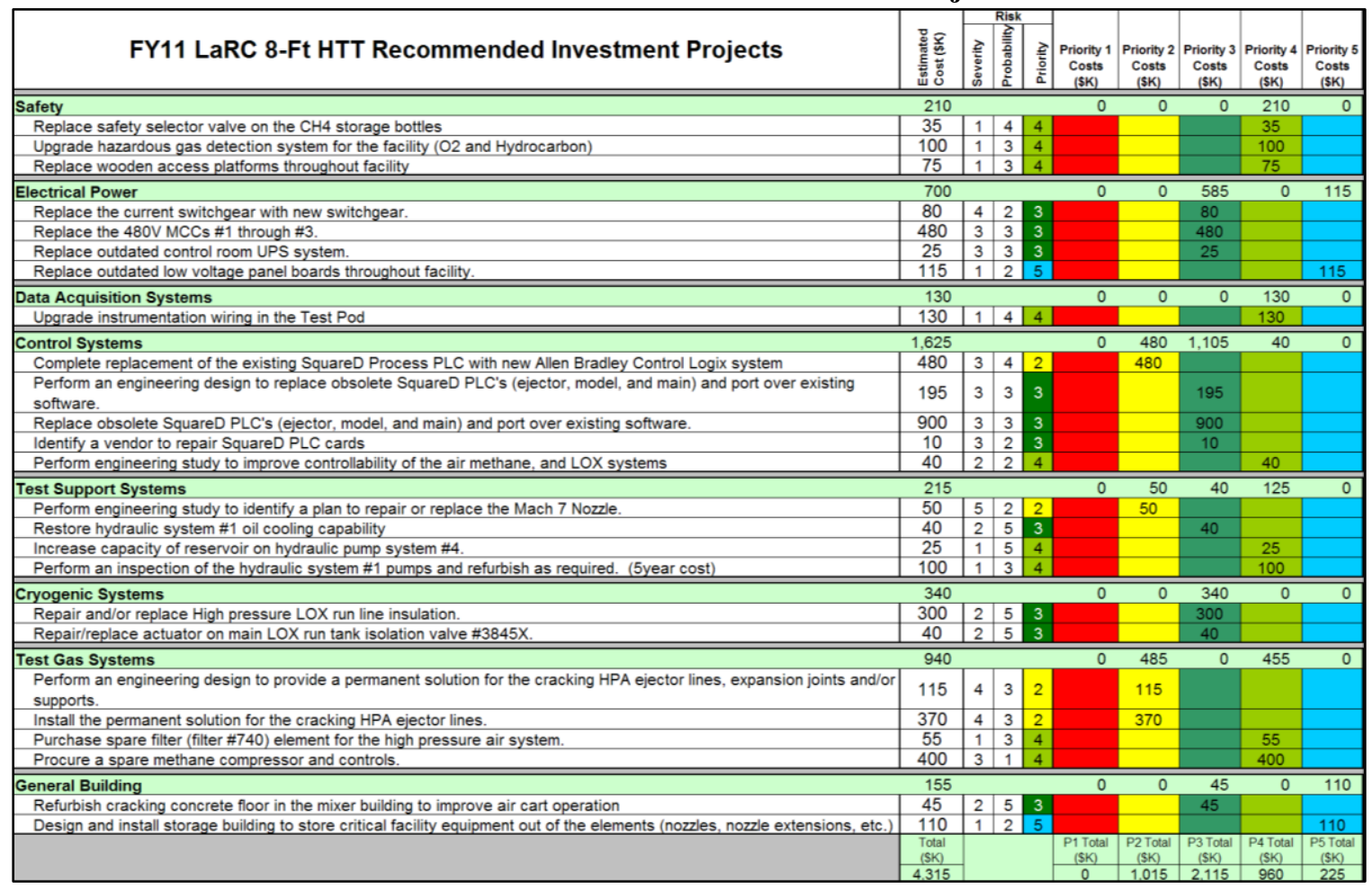

6

American Institute of Aeronautics and Astronautics 
ROM Cost Estimates: ROM cost estimates were prepared for each recommended project and included in the project database. An example of the LaRC 8-Ft HTT project estimates is presented in Table 4.

Table 4. FY11 LaRC 8-Ft HTT Assessment Project ROM Cost Estimates

\begin{tabular}{|c|c|c|c|c|c|c|c|c|c|c|}
\hline LaRC 8' HTT Recommendations & $\begin{array}{c}\text { FY11 } \\
\text { Estimated } \\
\text { Cost }\end{array}$ & Details & $\begin{array}{l}\text { Labor } \\
\text { Hours }\end{array}$ & $\begin{array}{c}\text { Eng } \\
\text { Hours }\end{array}$ & & $\begin{array}{l}\text { Hours } \\
\text { Total }\end{array}$ & & terials/ & & Total \\
\hline $\begin{array}{l}\text { Upgrade hazardous gas detection system for the facility (O2 and } \\
\text { Hydrocarbon) }\end{array}$ & 100 & $\begin{array}{l}\text { Engineering }=200 \mathrm{mhrs}(10 \text { drawings } @ 20 \mathrm{hrs} / \text { sheet }) \\
\text { Installation }=320 \mathrm{mhrs}(2 \text { techs } 1 \text { month }) \\
\text { Commissioning }=80 \mathrm{mhrs} \\
\text { Material }=\$ 30,000\end{array}$ & 320 & 280 & $\$$ & 65,600 & $\$$ & 30,000 & $\$$ & 95,600 \\
\hline Replace wooden access platforms throughout facility & 75 & $\begin{array}{l}\text { Assume: } 3 \text { platforms affected. Note given that designs for the new } \\
\text { platforms are already complete. Assume some type modular component } \\
\text { design, no excavation, concrete work, etc required. } \\
\text { Engineering }=160 \text { mhrs support } \\
\text { Installation }=240 \text { mhrs ( } 2 \text { craft, } 1 \text { week/platform) } \\
\text { Material }=\$ 30 \mathrm{~K} \text { ( } \$ 10 \mathrm{~K} \text { platform) }\end{array}$ & 240 & 160 & $\$$ & 43,200 & $\$$ & 30,000 & $\$$ & 73,200 \\
\hline Replace safety selector valve on the $\mathrm{CH} 4$ storage bottles & 35 & $\begin{array}{l}\text { Assume } 500 \text { psi system, and that } \mathrm{CH} 4 \text { must be removed for tanks to } \\
\text { install new valve } \\
\text { Valve }=\$ 10 \mathrm{~K} \\
\text { Eng }=80 \mathrm{Mhrs} \\
\text { Labor }=120 \text { mhrs (crew of } 3 \text { for } 1 \text { week) }\end{array}$ & 120 & 80 & $\$$ & 21,600 & $\$$ & 10,000 & $\$$ & 31,600 \\
\hline Replace the current switchgear with new switchgear. & 80 & $\begin{array}{l}\text { Engineering }=80 \text { mhrs } \\
\text { Labor }=300 \mathrm{mhrs} \\
\text { Material }=\$ 40,000\end{array}$ & 300 & 80 & $\$$ & 39,600 & $\$$ & 40,000 & $\$$ & 79,600 \\
\hline Replace the $480 \mathrm{~V}$ MCCs $\# 1$ through \#3. & 480 & $\begin{array}{l}\text { Engineering }=160 \mathrm{mhrs} \\
\text { Labor }=600 \mathrm{mhrs} \\
\text { Material }=\$ 400,000\end{array}$ & 600 & 160 & $\$$ & 79,200 & $\$$ & 400,000 & $\$$ & 479,200 \\
\hline Replace outdated low voltage panel boards throughout facility. & 115 & $\begin{array}{l}\text { Engineering }=120 \mathrm{mhrs} \\
\text { Labor }=400 \mathrm{mhrs} \\
\text { Material }=\$ 60,000\end{array}$ & 400 & 120 & $\$$ & 54,400 & $\$$ & 60,000 & $\$$ & 114,400 \\
\hline Replace outdated control room UPS system. & 25 & $\begin{array}{l}\text { Assume: } 18 \text { kVA UPS } \\
\text { Engineering }=40 \mathrm{mhrs} \\
\text { Labor }=32 \mathrm{mhrs} \\
\text { Materials }=\$ 15,000\end{array}$ & 32 & 40 & $\$$ & 8,000 & $\$$ & 15,000 & $\$$ & 23,000 \\
\hline Upgrade instrumentation wiring in the Test Pod & 130 & $\begin{array}{l}\text { Engineering (Design and Construction support })=160 \text { mhrs } \\
\text { Labor }=960 \text { mhrs }\left(2 \text { techs }{ }^{*} 3 \text { months }\right) \\
\text { Materials }=\$ 10,000\end{array}$ & 960 & 160 & $\$$ & 115,200 & $\$$ & 10,000 & $\$$ & 125,200 \\
\hline $\begin{array}{l}\text { Perform an engineering design to replace obsolete SquareD } \\
\text { PLC's (ejector, model, and main) and port over existing } \\
\text { software. }\end{array}$ & 195 & $\begin{array}{l}\text { Engineering }=800 \mathrm{mhrs}(50 \text { drawings @ } 16 \mathrm{hrs} / \mathrm{dwg}) \\
\text { Engineering }=200 \mathrm{mhrs} \text { (Software design) } \\
\text { Engineering }=600 \mathrm{mhrs} \text { (Software development, } 200 \mathrm{mhrs} / \mathrm{PLC} \text { ) }\end{array}$ & & 1600 & $\$$ & 192,000 & & & $\$$ & 192,000 \\
\hline $\begin{array}{l}\text { Replace obsolete SquareD PLC's (ejector, model, and main) } \\
\text { and port over existing software. }\end{array}$ & 900 & $\begin{array}{l}\text { Engineering }=600 \text { mhrs (Software checkout } 200 \text { mhrs } / \text { PLC) } \\
\text { Labor }=480 \text { mhrs (lnstallation \& checkout, } 160 \text { mhrs } / \text { PLC) } \\
\text { Commissioning }=\$ 720 \mathrm{~K}(1 \text { month@ } \$ 60 \mathrm{k} / \text { week } \times 3 \text { PLCs) } \\
\text { Misc Mtrls }=\$ 60 \mathrm{~K} \text { (\$20k/PLC) }\end{array}$ & 480 & 600 & $\$$ & 120,000 & $\$$ & 780,000 & $\$$ & 900,000 \\
\hline $\begin{array}{l}\text { Complete replacement of the existing SquareD Process PLC } \\
\text { with new Allen Bradley Control Logix system }\end{array}$ & 480 & $\begin{array}{l}\text { Assume Design complete } \\
\text { Engineering }=200 \text { mhrs (Software checkout) } \\
\text { Labor }=160 \text { mhrs (llnstallation } \& \text { checkout, } 160 \text { mhrs/PLC) } \\
\text { Commissioning }=480 \mathrm{~K} \text { ( } 2 \text { month@ } \$ 60 \mathrm{k} / \text { week -NASA provided cost) } \\
\text { Misc Mtrls }=\$ 10 \mathrm{~K}\end{array}$ & 160 & 200 & $\$$ & 40,000 & $\$$ & 250,000 & $\$$ & 480,000 \\
\hline dentify a vendor to repair SquareD PLC cards & 10 & Engineering $=80 \mathrm{mhrs}$ & & 80 & $\$$ & 9,600 & & & $\$$ & 9,600 \\
\hline
\end{tabular}

\section{Overall Assessment Results}

While there are many ways the assessment project data can be sorted and viewed to identify trends, several revealing views are presented and discussed in the following sections.

\section{A. FY08 Assessment Project Status}

During the FY11 assessment, the assessment team reviewed the status of the projects identified in the FY08 assessment. A status was assigned to each assessment project. The status categories were:

Completed: Project has been completed and closed out

In Progress: Project has been funded but not yet completed at the time of the assessment

Removed/Replaced: It was determined that the project was no longer needed or was included in a new project

Planned: FY08 projects that have not been completed, removed, or are in progress

New: Project was added during the FY11 assessment 
The total number of projects for each status category is presented in Table 5 and Fig.5. From this data, it can be seen that a significant percentage of the FY08 projects (32\% average) were removed or replaced. The majority of removed projects were replaced or combined with new projects that better address the facility needs while the remainder was removed because they were no longer required due to changes in facility needs since the FY08 assessment. On average, 25\% of all FY08 facility projects were completed or are funded and in progress. 400 new projects were identified during the FY11 assessment.

Table 5. FY08 Assessment Project Status.

\begin{tabular}{|r|c|c|c|c|c|c|}
\hline ATP Facilities & $\begin{array}{c}\text { FY08 } \\
\text { Total } \\
\text { Projects }\end{array}$ & $\begin{array}{c}\text { Projects } \\
\text { Completed } \\
\text { or In Progress }\end{array}$ & $\begin{array}{c}\text { Removed/ } \\
\text { Replaced } \\
\text { Projects }\end{array}$ & $\begin{array}{c}\text { \% Completed } \\
\text { or In Progress }\end{array}$ & $\begin{array}{c}\text { \% Projects } \\
\text { Removed/ } \\
\text { Replaced }\end{array}$ & $\begin{array}{c}\text { FY11 } \\
\text { New } \\
\text { Projects }\end{array}$ \\
\hline ARC 11Ft \& 9x7 & 78 & 17 & 36 & $22 \%$ & $46 \%$ & 101 \\
\hline GRC - IRT & 23 & 6 & 11 & $26 \%$ & $48 \%$ & 26 \\
\hline GRC - PSL & 31 & 8 & 3 & $26 \%$ & $10 \%$ & 25 \\
\hline GRC - 8x6-9x15 & 45 & 17 & 15 & $38 \%$ & $33 \%$ & 33 \\
\hline GRC - 10x10 & 39 & 8 & 14 & $21 \%$ & $36 \%$ & 47 \\
\hline LaRC - LaRC - NTF & 50 & 12 & 12 & $24 \%$ & $24 \%$ & 52 \\
\hline LaRC - TDT & 69 & 19 & 24 & $28 \%$ & $35 \%$ & 27 \\
\hline LaRC - 14x22 & 28 & 7 & 10 & $25 \%$ & $36 \%$ & 27 \\
\hline LaRC - 8ft HTT & 22 & 4 & 8 & $18 \%$ & $36 \%$ & 15 \\
\hline LaRC - 31" M10 & 33 & 12 & 10 & $36 \%$ & $30 \%$ & 35 \\
\hline LaRC - 20" M6 & 23 & 4 & 5 & $17 \%$ & $22 \%$ & 12 \\
\hline
\end{tabular}

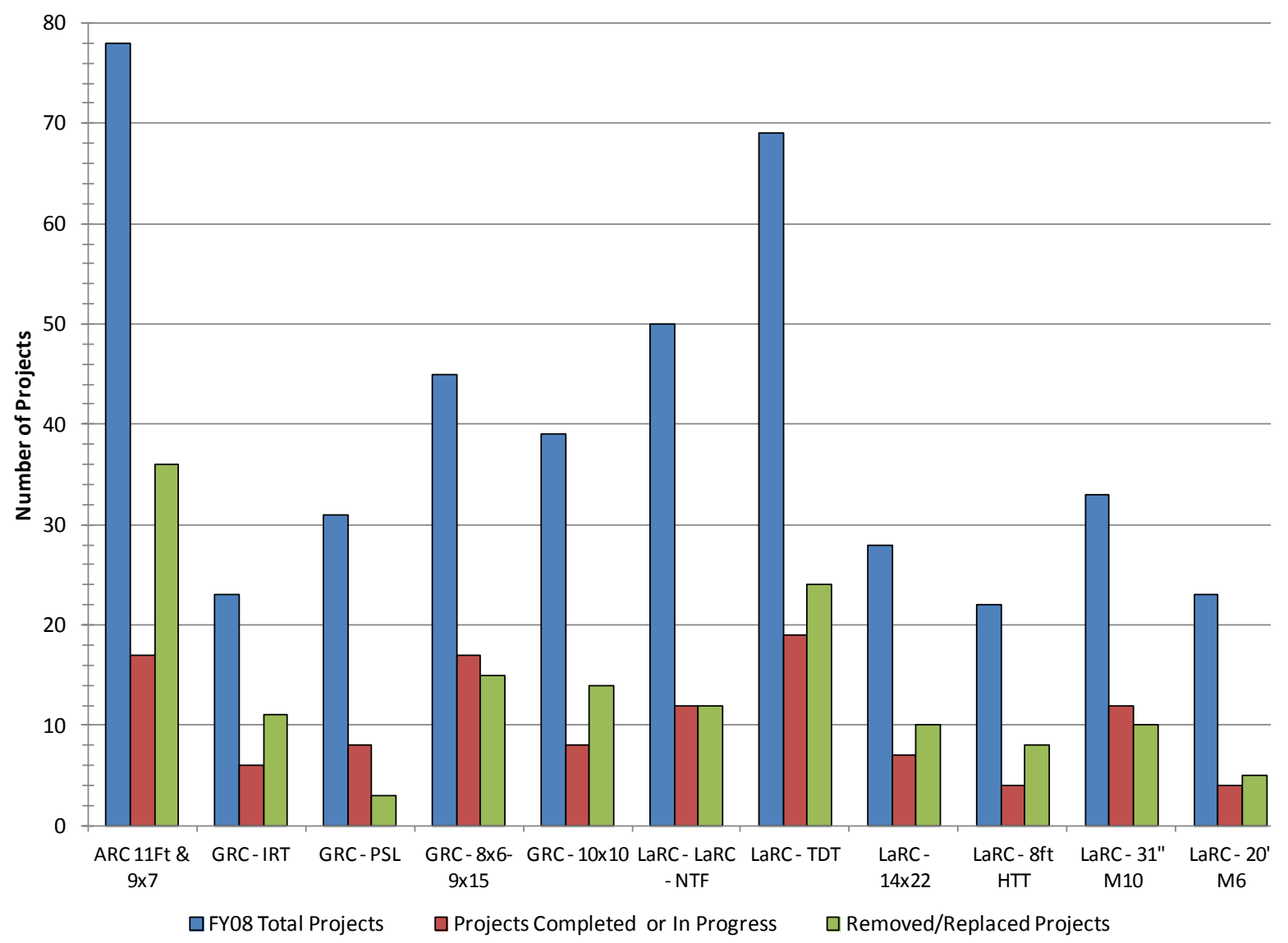

Figure 5. FY08 Assessment Project Status 


\section{B. Summary of Investment Costs by Facility and Priority}

A summary of the total recommended project costs for all assessed facilities are presented in Tables 6,7 , and 8 and Fig. 6. Approximately $\$ 185 \mathrm{M}$ of investment projects were recommended to be accomplished over the next five years.

Table 6. FY11 ATP Project Costs by Facility and Priority (\$M)

\begin{tabular}{|r|c|c|c|c|c|c|c|}
\hline \multicolumn{1}{|c|}{ ATP Facilities } & $\begin{array}{c}\text { Priority } \\
\mathbf{1}\end{array}$ & $\begin{array}{c}\text { Priority } \\
\mathbf{2}\end{array}$ & $\begin{array}{c}\text { Priority } \\
\mathbf{3}\end{array}$ & $\begin{array}{c}\text { Priority } \\
\mathbf{4}\end{array}$ & $\begin{array}{c}\text { Priority } \\
\mathbf{5}\end{array}$ & $\begin{array}{c}\text { Facility } \\
\text { Total }\end{array}$ & $\begin{array}{c}\text { Facility Total/ } \\
\text { Total Cost }\end{array}$ \\
\hline ARC 11' \& 9x7 WT & 0.04 & 9.85 & 16.43 & 27.81 & 0.13 & $\mathbf{5 4 . 2 5}$ & $\mathbf{2 9 . 3 \%}$ \\
\hline LaRC NTF & 0.15 & 1.92 & 9.53 & 11.72 & 0.15 & $\mathbf{2 3 . 4 6}$ & $\mathbf{1 2 . 7 \%}$ \\
\hline LaRC 14x22 WT & 0.00 & 0.81 & 12.30 & 9.73 & 0.00 & $\mathbf{2 2 . 8 3}$ & $\mathbf{1 2 . 3 \%}$ \\
\hline GRC 10x10 WT & 0.00 & 0.40 & 6.12 & 10.73 & 0.66 & $\mathbf{1 7 . 9 0}$ & $\mathbf{9 . 7 \%}$ \\
\hline LaRC TDT & 0.16 & 0.26 & 4.18 & 11.28 & 0.01 & $\mathbf{1 5 . 8 8}$ & $\mathbf{8 . 6 \%}$ \\
\hline GRC IRT & 0.00 & 3.01 & 3.87 & 6.22 & 0.37 & $\mathbf{1 3 . 4 7}$ & $\mathbf{7 . 3 \%}$ \\
\hline GRC PSL & 0.34 & 0.78 & 5.54 & 3.19 & 0.95 & $\mathbf{1 0 . 7 8}$ & $\mathbf{5 . 8 \%}$ \\
\hline GRC 8x6 \& 9x15 TWT & 0.00 & 1.57 & 2.08 & 4.92 & 0.95 & $\mathbf{9 . 5 2}$ & $\mathbf{5 . 1 \%}$ \\
\hline LaRC 20" M6 WT & 1.14 & 0.17 & 1.00 & 5.80 & 0.57 & $\mathbf{7 . 5 4}$ & $\mathbf{4 . 1 \%}$ \\
\hline LaRC 8' HTWT & 0.00 & 1.02 & 2.12 & 0.96 & 0.23 & $\mathbf{4 . 3 2}$ & $\mathbf{2 . 3 \%}$ \\
\hline Priority Total & $\mathbf{1 . 8 2}$ & $\mathbf{1 9 . 9 3}$ & $\mathbf{6 4 . 0 5}$ & $\mathbf{9 5 . 0 3}$ & $\mathbf{4 . 1 6}$ & $\mathbf{1 8 4 . 9 9}$ & $\mathbf{1 0 0 . 0 \%}$ \\
\hline Priority Total/Total Cost & $\mathbf{1 . 0} \%$ & $\mathbf{1 0 . 8 \%}$ & $\mathbf{3 4 . 6 \%}$ & $\mathbf{5 1 . 4 \%}$ & $\mathbf{2 . 2} \%$ & $\mathbf{1 0 0 . 0} \%$ &
\end{tabular}

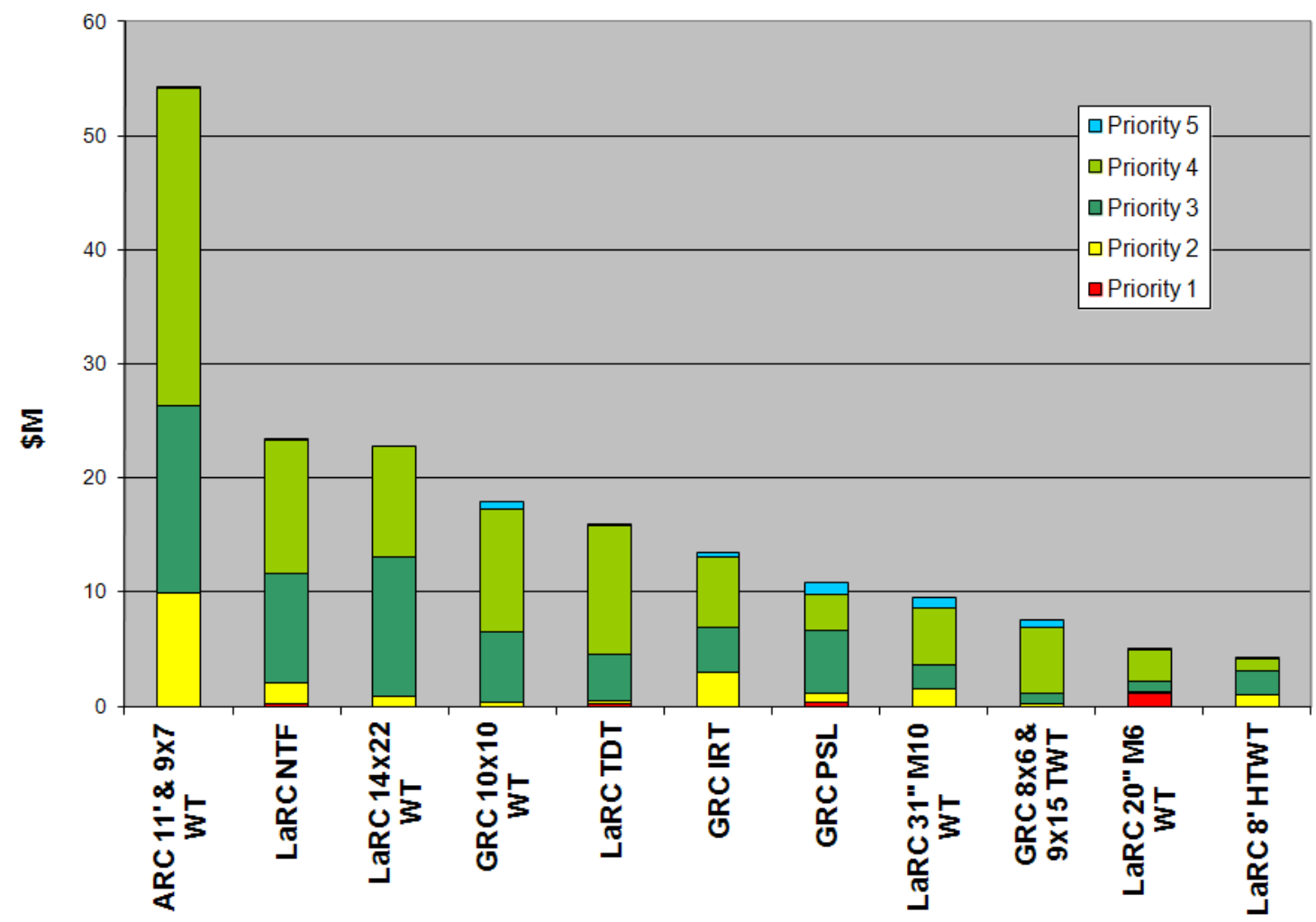

Figure 6. FY11 ATP Facilities Project Costs by Facility 
Table 7. FY11 ATP Project Costs Percentages by Facility ( $\%$ of Priority Total)

\begin{tabular}{|r|c|c|c|c|c|}
\hline \multicolumn{1}{|c|}{ ATP Facilities } & Priority & Priority & Priority & Priority & Priority \\
\hline ARC 11' \& 9x7 WT & $0.0 \%$ & $15.1 \%$ & $6.0 \%$ & $6.5 \%$ & 5 \\
\hline LaRC NTF & $18.5 \%$ & $3.9 \%$ & $8.6 \%$ & $3.4 \%$ & $22.8 \%$ \\
\hline LaRC 14x22 WT & $0.0 \%$ & $2.0 \%$ & $9.6 \%$ & $11.3 \%$ & $15.7 \%$ \\
\hline GRC 10x10 WT & $0.0 \%$ & $0.8 \%$ & $1.6 \%$ & $6.1 \%$ & $13.7 \%$ \\
\hline LaRC TDT & $2.2 \%$ & $49.4 \%$ & $25.6 \%$ & $29.3 \%$ & $3.1 \%$ \\
\hline GRC IRT & $8.5 \%$ & $1.3 \%$ & $6.5 \%$ & $11.9 \%$ & $0.2 \%$ \\
\hline GRC PSL & $0.0 \%$ & $4.0 \%$ & $19.2 \%$ & $10.2 \%$ & $0.0 \%$ \\
\hline LaRC 31" M10 WT & $8.0 \%$ & $9.6 \%$ & $14.9 \%$ & $12.3 \%$ & $3.5 \%$ \\
\hline GRC 8x6 \& 9x15 TWT & $0.0 \%$ & $5.1 \%$ & $3.3 \%$ & $1.0 \%$ & $5.4 \%$ \\
\hline LaRC 20" M6 WT & $0.0 \%$ & $7.9 \%$ & $3.2 \%$ & $5.2 \%$ & $22.8 \%$ \\
\hline LaRC 8' HTWT & $62.8 \%$ & $0.9 \%$ & $1.4 \%$ & $2.8 \%$ & $3.7 \%$ \\
\hline ATP Priority/Total & $\mathbf{1 0 0 . 0 \%}$ & $\mathbf{1 0 0 . 0} \%$ & $\mathbf{1 0 0 . 0} \%$ & $\mathbf{1 0 0 . 0} \%$ & $\mathbf{1 0 0 . 0 \%}$ \\
\hline
\end{tabular}

Table 8. FY11 ATP Project Costs Percentages by Facility (\% of Facility Total)

\begin{tabular}{|c|c|c|c|c|c|c|}
\hline ATP Facilities & $\begin{array}{c}\text { Priority } \\
1\end{array}$ & $\begin{array}{c}\text { Priority } \\
2\end{array}$ & $\begin{array}{c}\text { Priority } \\
3\end{array}$ & $\begin{array}{c}\text { Priority } \\
4\end{array}$ & $\begin{array}{c}\text { Priority } \\
5\end{array}$ & $\begin{array}{l}\text { Fac } \\
\text { Total }\end{array}$ \\
\hline ARC 11' \& 9x7 WT & $0.0 \%$ & $22.4 \%$ & $28.7 \%$ & $46.2 \%$ & $2.7 \%$ & $100.0 \%$ \\
\hline LaRC NTF & $3.1 \%$ & $7.2 \%$ & $51.3 \%$ & $29.5 \%$ & $8.8 \%$ & $100.0 \%$ \\
\hline LaRC 14x22 WT & $0.0 \%$ & $2.2 \%$ & $34.2 \%$ & $59.9 \%$ & $3.7 \%$ & $100.0 \%$ \\
\hline GRC 10x10 WT & $0.0 \%$ & $2.2 \%$ & $13.3 \%$ & $77.0 \%$ & $7.6 \%$ & $100.0 \%$ \\
\hline LaRC TDT & $0.1 \%$ & $18.2 \%$ & $30.3 \%$ & $51.3 \%$ & $0.2 \%$ & $100.0 \%$ \\
\hline GRC IRT & $1.0 \%$ & $1.6 \%$ & $26.3 \%$ & $71.1 \%$ & $0.1 \%$ & $100.0 \%$ \\
\hline GRC PSL & $0.0 \%$ & $3.5 \%$ & $53.9 \%$ & $42.6 \%$ & $0.0 \%$ & $100.0 \%$ \\
\hline LaRC 31" M10 WT & $0.6 \%$ & $8.2 \%$ & $40.6 \%$ & $50.0 \%$ & $0.6 \%$ & $100.0 \%$ \\
\hline GRC 8x6 \& 9x15 TWT & $0.0 \%$ & $23.5 \%$ & $49.0 \%$ & $22.2 \%$ & $5.2 \%$ & $100.0 \%$ \\
\hline LaRC 20" M6 WT & $0.0 \%$ & $16.5 \%$ & $21.8 \%$ & $51.7 \%$ & $10.0 \%$ & $100.0 \%$ \\
\hline LaRC 8' HTWT & $22.5 \%$ & $3.5 \%$ & $18.0 \%$ & $53.0 \%$ & $3.1 \%$ & $100.0 \%$ \\
\hline Average & $2.5 \%$ & $9.9 \%$ & $33.4 \%$ & $50.4 \%$ & $3.8 \%$ & $100.0 \%$ \\
\hline
\end{tabular}

The data presented in these tables and figure provides a high level look at the total recommended investment costs sorted by facility and their assigned priority. While this data can be analyzed in many different ways, several trends were identified (percentages given as \% of total costs).

- The total recommended investment cost is $\$ 185 \mathrm{M}$.

- $1.0 \%(\$ 1.82 \mathrm{M})$ of the recommendations are Priority $1-$ most urgent.

- $10.8 \%(\$ 19.93 \mathrm{M})$ of the recommendations are Priority 2 - urgent.

- $34.6 \%(\$ 64.1 \mathrm{M})$ of the recommendations are Priority 3.

- $51.4 \%(95 \mathrm{M})$ of the recommendations are Priority 4

- The LaRC 20" M6 tunnel accounts for 63\% (\$1.14M) of the Priority 1 project costs. This is a control systems project to replace the model attitude and model injection system which is obsolete and has chronic failures. (see Section 5.10 for project details).

- The ARC $11 \mathrm{Ft} / 9 \times 7$ requires the largest investment $(\$ 54.3 \mathrm{M}$ or $29.3 \%)$ of all the assessed facilities.

- The ARC 11Ft/9x7 data acquisition systems projects account for $44 \%(\$ 24.2 \mathrm{M})$ of these costs. The $11 \mathrm{Ft} / 9 \times 7$ facilities has a comprehensive five-year DAS upgrade plan to address continuous improvements to their systems. This upgrade plan involves the phased replacement of selected obsolete hardware elements of the systems with functionally equivalent elements based on current technologies and the modification of the supporting software for the replaced elements. All of the projects from this five year plan were included in the FY11 projects database. 
- The 11Ft/9x7 facility costs also comprise 49\% (\$9.9M) of all the Priority 2 project costs. These projects fall primarily under the electrical power (replacement of capacitor banks and medium voltage MCC's) and test facility structures (Seismic upgrade to MUA and HPA piping supports) categories. See Section 5.1 for project details.

- The LaRC NTF ( $\$ 23.5 \mathrm{M}$ or $12.7 \%)$ and the LaRC $14 \times 22$ ( $\$ 22.8$ or $12.3 \%)$ are the second and third largest investment costs respectively.

- While on average, the majority of the total costs fall under Priority 3 and Priority 4 projects, almost one quarter of the GRC IRT $(\$ 3 \mathrm{M})$ and the LaRC 8-Ft HTT $(\$ 1 \mathrm{M})$ total project costs are Priority 2 projects. The IRT projects primarily address the facility fan/compressor systems (replace the man fan variable frequency drive system and procure spare fan blades). The 8-Ft HTT projects address the control systems (e.g., replace the obsolete main, ejector, and model control PLCs)

- The Priority 3 and Priority 4 projects comprise approximately $83 \%$ of the total project costs (Priority 3 $33.4 \%$ and Priority $4-50.4 \%$ )

\section{Summary of Investment Costs by Facility System Category and Priority}

The data sorts presented in this section provide a view of the project priorities in relation to facility system categories. These views allow one to trend the recommended project costs for each facility system and their associated priorities. Tables 9, 10, 11, and 12, and Figure 7 presents the recommended project costs for the ATP facilities sorted by facility system category and priority.

Table 9. FY11 ATP Facilities Project Costs by System Category (\$M)

\begin{tabular}{|c|c|c|c|c|c|c|c|}
\hline System Category & $\begin{array}{c}\text { Priority } \\
1\end{array}$ & \begin{tabular}{|c|} 
Priority \\
2
\end{tabular} & $\begin{array}{c}\text { Priority } \\
3\end{array}$ & $\begin{array}{c}\text { Priority } \\
4\end{array}$ & $\begin{array}{c}\text { Priority } \\
5\end{array}$ & $\begin{array}{l}\text { Cat } \\
\text { Total }\end{array}$ & $\begin{array}{l}\text { Cat Total/ } \\
\text { Total Cost }\end{array}$ \\
\hline Safety & 0.16 & 0.65 & 0.51 & 0.61 & 0.00 & 1.92 & $1.0 \%$ \\
\hline Electrical Power & 0.34 & 4.61 & 10.37 & 8.01 & 0.33 & 23.64 & $12.8 \%$ \\
\hline Data Acquisition Systems & 0.00 & 0.12 & 20.85 & 35.70 & 0.13 & 56.79 & $30.7 \%$ \\
\hline Control Systems & 1.24 & 2.79 & 3.49 & 9.56 & 1.89 & 18.96 & $10.2 \%$ \\
\hline Test Facility Structures & 0.00 & 6.14 & 2.98 & 17.14 & 0.56 & 26.81 & $14.5 \%$ \\
\hline Cooling Water Systems & 0.00 & 0.47 & 12.16 & 1.13 & 0.00 & 13.75 & $7.4 \%$ \\
\hline Test Support Systems & 0.09 & 1.55 & 7.61 & 8.22 & 0.83 & 18.29 & $9.9 \%$ \\
\hline Cryogenic Systems & 0.00 & 0.00 & 1.66 & 0.92 & 0.04 & 2.61 & $1.4 \%$ \\
\hline Vacuum Systems & 0.00 & 0.00 & 0.03 & 0.00 & 0.00 & 0.03 & $0.0 \%$ \\
\hline Test Gas Systems & 0.00 & 0.97 & 3.03 & 8.05 & 0.26 & 12.30 & $6.6 \%$ \\
\hline Facility Fan/Compressor Systems & 0.00 & 2.64 & 0.70 & 4.06 & 0.00 & 7.40 & $4.0 \%$ \\
\hline General Building & 0.00 & 0.00 & 0.70 & 1.66 & 0.14 & 2.50 & $1.3 \%$ \\
\hline Priority Total & 1.82 & 19.93 & 64.05 & 95.03 & 4.16 & 184.99 & $100.0 \%$ \\
\hline Priority Total/Total Cost & $1.0 \%$ & $10.8 \%$ & $34.6 \%$ & $51.4 \%$ & $2.2 \%$ & $100.0 \%$ & \\
\hline
\end{tabular}




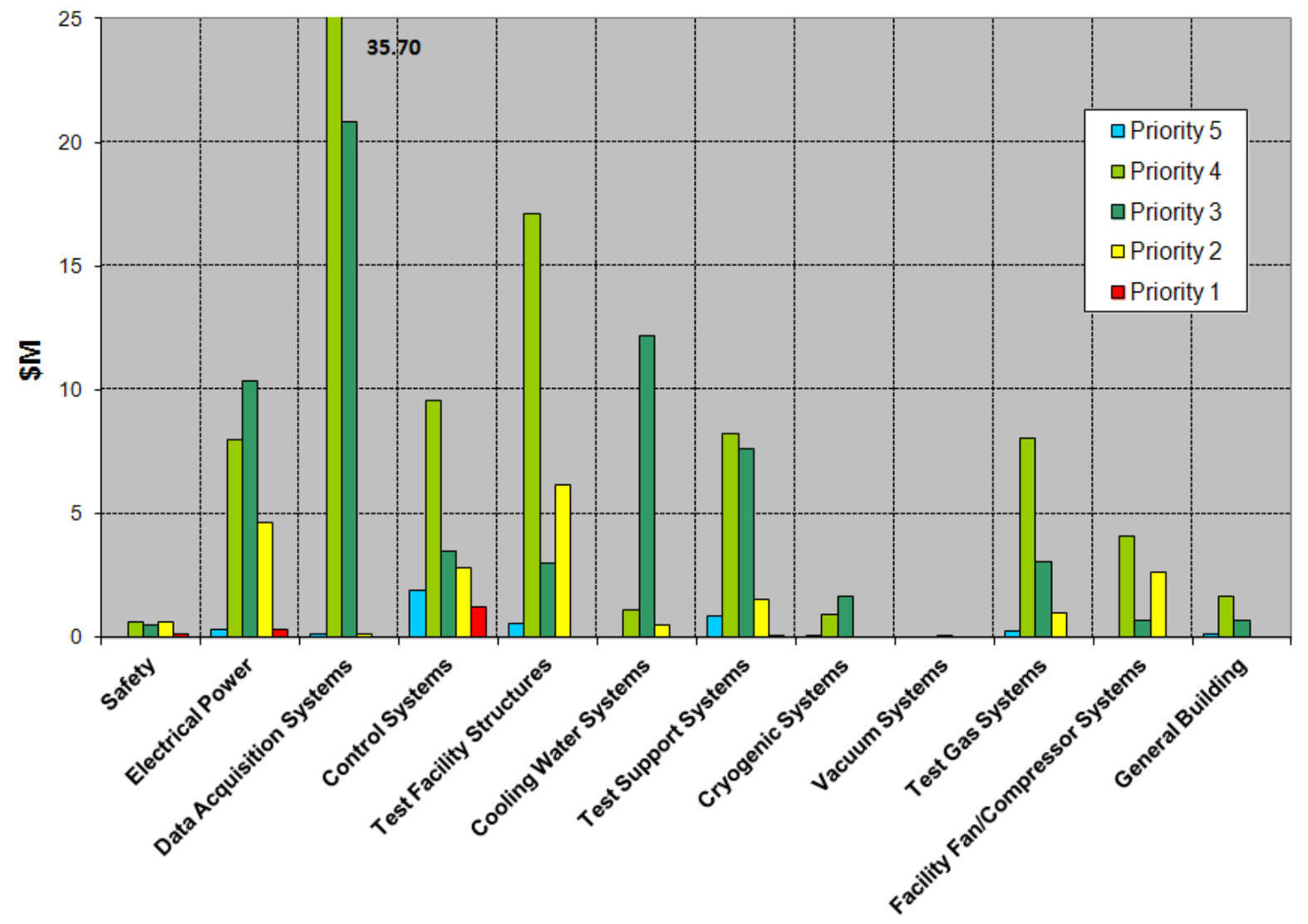

Figure 7. FY11 ATP Facilities Investment Costs by System Category

Table 10. FY11 ATP Project Costs Percentages by System Category (\% of Priority Total)

\begin{tabular}{|r|c|c|c|c|c|}
\hline System Category & Priority & Priority & Priority & Priority & Priority \\
\hline Safety & $\mathbf{1}$ & $\mathbf{2}$ & $\mathbf{3}$ & $\mathbf{4}$ & $\mathbf{5}$ \\
\hline Electrical Power & $18.5 \%$ & $3.2 \%$ & $0.8 \%$ & $0.6 \%$ & $0.0 \%$ \\
\hline Data Acquisition Systems & $0.0 \%$ & $0.6 \%$ & $16.2 \%$ & $8.4 \%$ & $7.8 \%$ \\
\hline Control Systems & $68.0 \%$ & $14.0 \%$ & $5.4 \%$ & $37.6 \%$ & $3.0 \%$ \\
\hline Test Facility Structures & $0.0 \%$ & $30.8 \%$ & $4.6 \%$ & $18.0 \%$ & $45.4 \%$ \\
\hline Cooling Water Systems & $0.0 \%$ & $2.4 \%$ & $19.0 \%$ & $1.2 \%$ & $0.0 \%$ \\
\hline Test Support Systems & $5.0 \%$ & $7.8 \%$ & $11.9 \%$ & $8.6 \%$ & $20.0 \%$ \\
\hline Cryogenic Systems & $0.0 \%$ & $0.0 \%$ & $2.6 \%$ & $1.0 \%$ & $1.0 \%$ \\
\hline Vacuum Systems & $0.0 \%$ & $0.0 \%$ & $0.0 \%$ & $0.0 \%$ & $0.0 \%$ \\
\hline Test Gas Systems & $0.0 \%$ & $4.9 \%$ & $4.7 \%$ & $8.5 \%$ & $6.1 \%$ \\
\hline Facility Fan/Compressor Systems & $0.0 \%$ & $13.2 \%$ & $1.1 \%$ & $4.3 \%$ & $0.0 \%$ \\
\hline General Building & $0.0 \%$ & $0.0 \%$ & $1.1 \%$ & $1.7 \%$ & $3.4 \%$ \\
\hline Priority/Total & $\mathbf{1 0 0 . 0 \%}$ & $\mathbf{1 0 0 . 0 \%}$ & $\mathbf{1 0 0 . 0 \%}$ & $\mathbf{1 0 0 . 0} \%$ & $\mathbf{1 0 0 . 0 \%}$ \\
\hline
\end{tabular}


Table 11. FY11 ATP Project Costs Percentages by System Category (\% of Category Total)

\begin{tabular}{|r|c|c|c|c|c|c|}
\hline System Category & $\begin{array}{c}\text { Priority } \\
\mathbf{1}\end{array}$ & $\begin{array}{c}\text { Priority } \\
\mathbf{2}\end{array}$ & $\begin{array}{c}\text { Priority } \\
\mathbf{3}\end{array}$ & $\begin{array}{c}\text { Priority } \\
\mathbf{4}\end{array}$ & $\begin{array}{c}\text { Priority } \\
\mathbf{5}\end{array}$ & Total \\
\hline Safety & $8.1 \%$ & $33.7 \%$ & $26.6 \%$ & $31.6 \%$ & $0.0 \%$ & $\mathbf{1 0 0} \%$ \\
\hline Electrical Power & $1.4 \%$ & $19.5 \%$ & $43.8 \%$ & $33.9 \%$ & $1.4 \%$ & $\mathbf{1 0 0} \%$ \\
\hline Data Acquisition Systems & $0.0 \%$ & $0.2 \%$ & $36.7 \%$ & $62.9 \%$ & $0.2 \%$ & $\mathbf{1 0 0} \%$ \\
\hline Control Systems & $6.5 \%$ & $14.7 \%$ & $18.4 \%$ & $50.4 \%$ & $10.0 \%$ & $\mathbf{1 0 0} \%$ \\
\hline Test Facility Structures & $0.0 \%$ & $22.9 \%$ & $11.1 \%$ & $63.9 \%$ & $2.1 \%$ & $\mathbf{1 0 0} \%$ \\
\hline Cooling Water Systems & $0.0 \%$ & $3.4 \%$ & $88.4 \%$ & $8.2 \%$ & $0.0 \%$ & $\mathbf{1 0 0} \%$ \\
\hline Test Support Systems & $0.5 \%$ & $8.5 \%$ & $41.6 \%$ & $44.9 \%$ & $4.5 \%$ & $\mathbf{1 0 0} \%$ \\
\hline Cryogenic Systems & $0.0 \%$ & $0.0 \%$ & $63.4 \%$ & $35.1 \%$ & $1.5 \%$ & $\mathbf{1 0 0} \%$ \\
\hline Vacuum Systems & $0.0 \%$ & $0.0 \%$ & $100.0 \%$ & $0.0 \%$ & $0.0 \%$ & $\mathbf{1 0 0} \%$ \\
\hline Facility Fan/Compressor Systems & $0.0 \%$ & $7.9 \%$ & $24.6 \%$ & $65.4 \%$ & $2.1 \%$ & $\mathbf{1 0 0} \%$ \\
\hline General Building & $0.0 \%$ & $35.7 \%$ & $9.5 \%$ & $54.9 \%$ & $0.0 \%$ & $\mathbf{1 0 0} \%$ \\
\hline Average & $\mathbf{1 . 4 \%}$ & $\mathbf{1 2 . 0} \%$ & $28.1 \%$ & $66.3 \%$ & $5.6 \%$ & $\mathbf{1 0 0} \%$ \\
\hline & & & & & & \\
\hline
\end{tabular}

Table 12. FY11 ATP Facility Investment Costs by System Category (\$K)

\begin{tabular}{|c|c|c|c|c|c|c|c|c|c|c|c|c|c|}
\hline System Category & $\begin{array}{l}\text { GRC } \\
\text { IRT }\end{array}$ & $\begin{array}{l}\text { GRC } \\
\text { PSL }\end{array}$ & $\begin{array}{c}\text { GRC } \\
10 \times 10\end{array}$ & $\begin{array}{c}\text { GRC } \\
8 \times 6 \& \\
9 \times 15\end{array}$ & $\begin{array}{c}\text { ARC 11 } \\
\& 9 \times 7\end{array}$ & $\begin{array}{l}\text { LaRC } \\
\text { TDT }\end{array}$ & $\begin{array}{l}\text { LaRC } \\
14 \times 22\end{array}$ & $\begin{array}{c}\text { LaRC } \\
\text { NTF }\end{array}$ & $\begin{array}{c}\text { LaRC } \\
8 f t \\
\text { HTT }\end{array}$ & $\begin{array}{c}\text { LaRC } \\
31 \mathrm{M10}\end{array}$ & $\begin{array}{l}\text { LaRC } \\
20 \mathrm{M6}\end{array}$ & Cat Tota & $\begin{array}{l}\text { Cat Total/ } \\
\text { Total Cost }\end{array}$ \\
\hline Safety & 175 & & 345 & 340 & 40 & 325 & 85 & 160 & 210 & 235 & & 1,915 & $1.0 \%$ \\
\hline Electrical Power & 1,435 & 1,475 & 9,280 & 480 & 4,280 & 600 & 670 & 3,345 & 700 & 1,170 & 205 & 23,640 & $12.8 \%$ \\
\hline Data Acquisition Systems & 1,165 & 2,985 & 2,425 & 3,120 & 24,170 & 2,575 & 12,085 & 4,845 & 130 & 1,295 & 1,995 & 56,790 & $30.7 \%$ \\
\hline Control Systems & & 950 & 1,590 & 1,690 & 2,465 & 645 & 555 & 2,665 & 1,625 & 4,690 & 2,085 & 18,960 & $10.2 \%$ \\
\hline Test Facility Structures & 5,105 & 880 & 615 & 390 & 6,945 & 9,510 & 20 & 3,285 & & 60 & & 26,810 & $14.5 \%$ \\
\hline Cooling Water Systems & & 350 & 175 & & 12,585 & 70 & & 290 & & 280 & & 13,750 & $7.4 \%$ \\
\hline Test Support Systems & 2,690 & 1,290 & 2,340 & 400 & 1,665 & 1,015 & 1,205 & 6,215 & 215 & 780 & 475 & 18,290 & $9.9 \%$ \\
\hline Cryogenic Systems & & & & & & & & 2,270 & 340 & & & 2,610 & $1.4 \%$ \\
\hline Vacuum Systems & & & & & & 30 & & & & & & 30 & $0.0 \%$ \\
\hline Test Gas Systems & 80 & 2,200 & 815 & 100 & 945 & 270 & 6,000 & & 940 & 760 & 185 & 12,295 & $6.6 \%$ \\
\hline Fan/Compressor Systems & 2,775 & & 165 & 1,000 & 605 & 635 & 2,110 & 110 & & & & 7,400 & $4.0 \%$ \\
\hline General Building & 40 & 650 & 150 & 15 & 550 & 200 & 100 & 270 & 155 & 245 & 120 & 2,495 & $1.3 \%$ \\
\hline Facility Total & 13,465 & 10,780 & 17,900 & 7,535 & 54,250 & 15,875 & 22,830 & 23,455 & 4,315 & 9,515 & 5,065 & 184,985 & $100.0 \%$ \\
\hline Facility Total/Total Cost & $7.3 \%$ & $5.8 \%$ & $9.7 \%$ & $4.1 \%$ & $29.3 \%$ & $8.6 \%$ & $12.3 \%$ & $12.7 \%$ & $2.3 \%$ & $5.1 \%$ & $2.7 \%$ & $100.0 \%$ & \\
\hline
\end{tabular}


From the project cost data presented in the tables and figure above, the following can be concluded (percentages given as $\%$ of total costs):

- The controls system projects account for the largest Priority 1 project costs $(\$ 1.24 \mathrm{M}$ or $68 \%)$. These projects primarily address the LaRC 20 " M6 tunnel projects $(\$ 1.14 \mathrm{M})$

- The GRC PSL electrical systems project, to replace the $2.4 \mathrm{KV}$ main switchgear, accounts for $18.5 \%$ ( $\$ 340 \mathrm{~K}$ ) of the Priority 1 project costs

- The test facility structures projects account for the largest Priority 2 project costs $(\$ 6.14 \mathrm{M}$ or $31 \%)$. These projects primarily address ARC 11Ft/9x7 structural projects (Seismic upgrade to MUA and HPA piping supports).

- The electrical power category projects account for $23 \%(\$ 4.6 \mathrm{M})$ of the Priority 2 projects costs. These costs primarily address electrical power projects for the ARC $11 \mathrm{Ft} / 9 \times 7$ ( $\$ 3.3 \mathrm{M}$ to replace capacitor banks) and the LaRC 31" M10 (\$1.05M to replace heater supply SCR system).

- The control and facility fan/compressor systems project account for $14 \%(\$ 2.79 \mathrm{M})$ and $13.3 \%(\$ 2.64 \mathrm{M})$ of the Priority 2 project costs.

- $30.7 \%(\$ 56.8 \mathrm{M})$ of the total project costs address data acquisition system issues.

- $14.5 \%(\$ 26.8 \mathrm{M})$ of the total project costs address test facility structure issues.

- $12.8 \%(\$ 23.6 \mathrm{M})$ of the total project costs address electrical power issues.

- $10.2 \%(\$ 19 \mathrm{M})$ of the total project costs address control system issues.

- $9.9 \%(\$ 18.3 \mathrm{M})$ of the total project costs address test support system issues.

- $7.4 \%(\$ 13.8 \mathrm{M})$ of the total project costs address test gas system issues.

\section{References}

${ }^{1}$ Jacobs Technology, "FY11 Facility Assessment Study for Aeronautics Test Program, Final Report” May 2012. 